\title{
Simultaneous Optimization of Damping and Tracking Controller Parameters via Selective Pole Placement for Enhanced Positioning Bandwidth of Nanopositioners
}

\author{
Douglas Russell, Student Member, IEEE, Andrew J. Fleming, Member, IEEE \\ and Sumeet S. Aphale ${ }^{\dagger}$, Member, IEEE
}

\begin{abstract}
Positive Velocity and Position Feedback (PVPF) is a widely used control scheme in lightly damped resonant systems with collocated sensor actuator pairs. The popularity of PVPF is due to the ability to achieve a chosen damping ratio by repositioning the poles of the system. The addition of a necessary tracking controller causes the poles to deviate from the intended location and can be a detriment to the damping achieved. By designing the PVPF and tracking controllers simultaneously, the optimal damping and tracking can be achieved. Simulations show full damping of the first resonant mode whilst also achieving bandwidth greater than the natural frequency of the plant, allowing for high speed scanning with accurate tracking.
\end{abstract}

\section{INTRODUCTION}

Highly resonant systems suffer from harmonic excitation which can lead to performance degradation and even structural damage. Previous research suggests myriad of options to manage the behaviour of such systems. Theses generally fall into one of two categories: open- and closed-loop. Openloop control mechanisms are simple to implement and use as no sensing equipment is necessary. However, open-loop control is highly sensitive to perturbations in system dynamics, so a sufficiently accurate model is required. Closed-loop controllers, on the other hand, display excellent robustness making them the preferred choice [1].

A number of closed-loop damping controllers have been developed for such highly resonant systems, including Integral Resonant Control [2], Integral Force Feedback [3], Resonant Control [4], Positive Position Feedback [5] and Positive Velocity and Position Feedback (PVPF) [6]. These controllers have a wide range of different applications, including robotic manipulators [7], disk-drives [8], aircraft wings [9], nanopositioning stages [10], scanning probe microscopes [11], MEMS / NEMS [12] and high-density memory storage devices [13].

In this work, a nanopositioner will be used as an example system, though the proposed control design is equally applicable to other applications. Nanopositioning platforms have a dominant resonant mode at a relatively low frequency. The

D. Russell and S. S. Aphale are with the Centre for Applied Dynamics Research, School of Engineering, University of Aberdeen, UK.

A. J. Fleming is with the Centre for Complex Dynamic Systems and Control, School of Electrical Engineering and Computer Science, University of Newcastle, Australia.

$\dagger$ Corresponding author speed at which a nanopositioner can accurately trace a raster scan input is limited to approximately one hundredth of its dominant resonant frequency [14]. This presents a problem in applications requiring high scanning speeds.

Another major detriment to the performance of nanopositioning platforms is the nonlinear behaviour exhibited by the piezoelectric actuators. Hysteresis, creep and thermal drift can be observed during open-loop operation causing positioning errors. To minimize these errors some form of reference tracking is generally incorporated [15]. The use of an integral tracking controller to reduce positioning errors due to nonlinear effects has been experimentally verified in numerous works [4], [6], [10].

Traditionally, control schemes for nanopositioning are developed by first designing a damping controller to damp the first resonant mode of the system, and then adding a tracking controller and tuning its gain to achieve the best tracking performance. However, this methodology is flawed. The addition of the tracking controller causes the damped poles to move from their original desired location and alters the damping characteristics of the system. It is, therefore, imperative that the damping and tracking controllers are designed simultaneously to achieve the necessary damping and tracking performance.

\section{A. Objective}

The objective of this paper is to provide a method for choosing optimal controller parameters for positioning applications. The desired outcome is a closed-loop frequency response which exhibits a flat response at low frequencies and rolls off at higher frequencies. The reason for this is the major harmonic excitation, during scanning, is observed in the axis which receives a triangle wave input. A flat band response at low frequencies will preserve the harmonics which define the triangular shape, and the roll off of high frequencies will reduce the effects of higher order dynamics and noise.

\section{B. Structure of the paper}

The paper is structured as follows, Section II provides an overview of PVPF. Section III outlines the method for choosing the optimal controller parameters. This is based on a process of selective pole placement. In Section IV, simulations are provided to show the effectiveness of the 

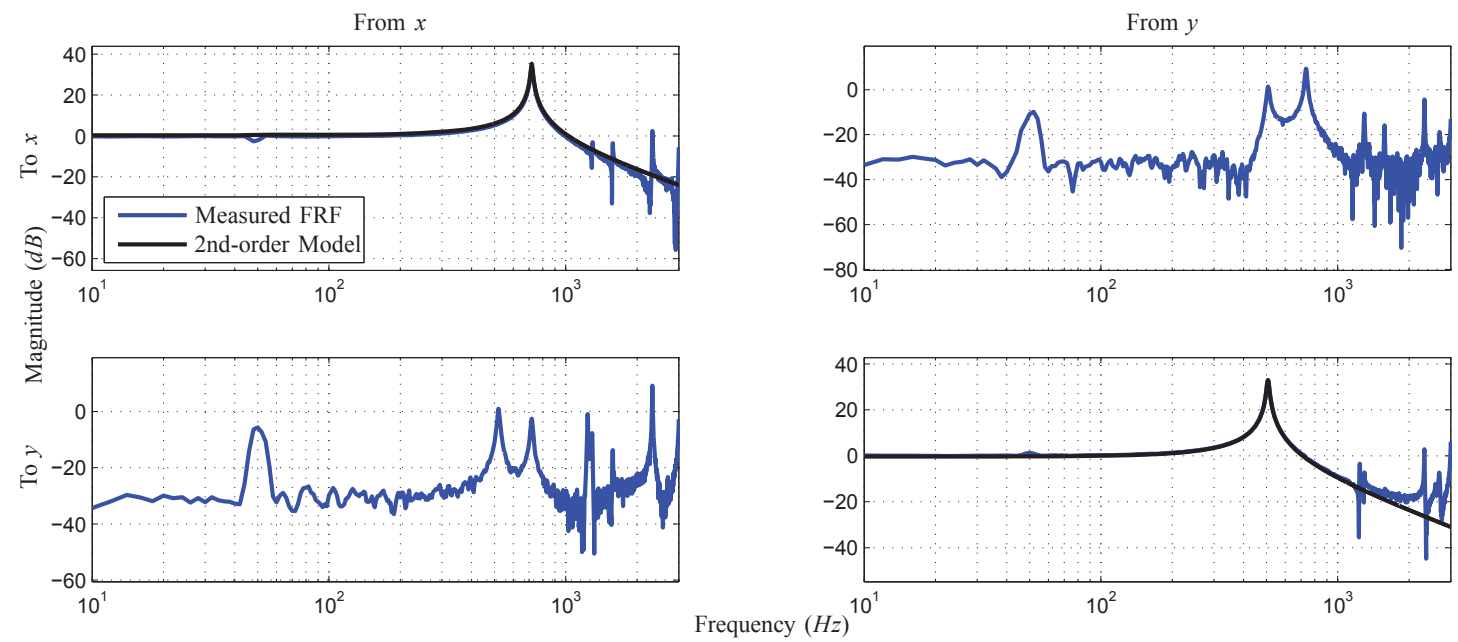

Fig. 1. Measured Frequency Response Fuction (FRF) of the nanopositioning platform (blue) and the derived second-order model (black). Higher-order models needed to capture the cross-coupling dynamics are not required for the control design and are not derived.

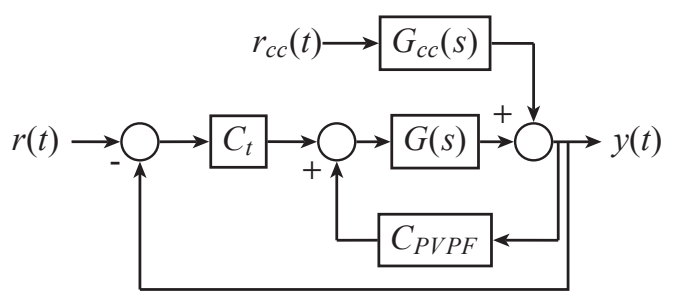

Fig. 2. Block diagram of the damped and tracked PVPF scheme, where $G(s)$ is the plant, $G_{c c}(s)$ is the FRF measuring the cross-coupling between the axes, $C_{P V P F}(s)$ is the damping controller, and $C_{t}(s)$ is the tracking controller.

optimal controller. This is based on the measured frequency response of a nanopositioning platform. Simulations are provided for both axes and includes cross-coupling between the axes. Section V concludes the paper.

\section{Positive Velocity and Position Feedback (PVPF)}

PVPF was introduced as an improvement on the existing PPF controller. Its design and implementation is similar to PPF with one exception: the addition of velocity feedback allows arbitrary pole placement, whereas PPF is limited in that regard. It has been shown to be an effective vibration damping controller in a nanopositioning applications [4], [6], [16]. The controller parameters are derived such that the damped system has two sets of complex poles at a specific location. Typically, this location is chosen by shifting the open-loop poles by an arbitrary, yet sufficiently large, amount into the left half plane. The frequency response of a nanopositioner measured from the applied voltage to the displacement can be represented by a second-order model as the first resonant mode is dominant, see Fig. 1. The model has transfer function

$$
G(s)=\frac{\sigma^{2}}{s^{2}+2 \zeta \omega_{n} s+\omega_{n}^{2}},
$$

where $\sigma^{2}$ corresponds to the DC gain, $\zeta$ is the damping ratio, and $\omega_{n}$ is the natural frequency. The PVPF controller has transfer function

$$
C_{P V P F}(s)=\frac{\Gamma_{2} s+\Gamma_{1}}{s^{2}+2 \gamma \omega_{c} s+\omega_{c}^{2}}
$$

A simple integrator is used as a tracking controller given by

$$
C_{t}(s)=\frac{k_{t}}{s} \text {. }
$$

The structure of the control scheme is shown in Fig. 2. As has been mentioned previously, the PVPF control scheme is designed by first choosing a damping controller to place the poles of the closed-loop system at a specified location. A tracking controller is then added and the gain is tuned to obtain the desired response. However, it has been shown that there is a relationship between the damping and tracking controllers and the stability of the system [17]. For this reason, the damping and tracking controllers will be designed simultaneously, i.e. only the damped and tracked system will be considered.

Denoting the numerator of a transfer function $G^{\text {num }}$, and the denominator $G^{\text {den }}$, such that $G=G^{\text {num }} / G^{\text {den }}$, the damped and tracked closed-loop system is given by

$$
\begin{array}{r}
G_{c l}^{n u m}(s)=k_{t} \sigma^{2}\left(s^{2}+2 \gamma \omega_{c}+\omega_{c}^{2}\right) \\
G_{c l}^{d e n}(s)=s^{5}+\left(2 \zeta \omega_{n}+2 \gamma \omega_{c}\right) s^{4}+\left(2 \zeta \omega_{n} 2 \gamma \omega_{c}+\omega_{n}^{2}+\omega_{c}^{2}\right) s^{3} \\
+\left(2 \zeta \omega_{n} \omega_{c}^{2}+2 \gamma \omega_{c} \omega_{n}^{2}-\sigma^{2} \Gamma_{2}+k_{t} \sigma^{2}\right) s^{2} \\
+\left(\omega_{n}^{2} \omega_{c}^{2}-\sigma^{2} \Gamma_{1}+k_{t} \sigma^{2} 2 \gamma \omega_{c}\right) s+k_{t} \sigma^{2} \omega_{c}^{2}
\end{array}
$$

This is used in the following section to derive the parameters of the proposed controller.

\section{Proposed Controller Design}

The traditional PVPF control scheme places the poles of the damped system at a specific location. As the tracking controller is implemented and the gain increased, the poles diverge from the chosen location. In this work, the opposite 

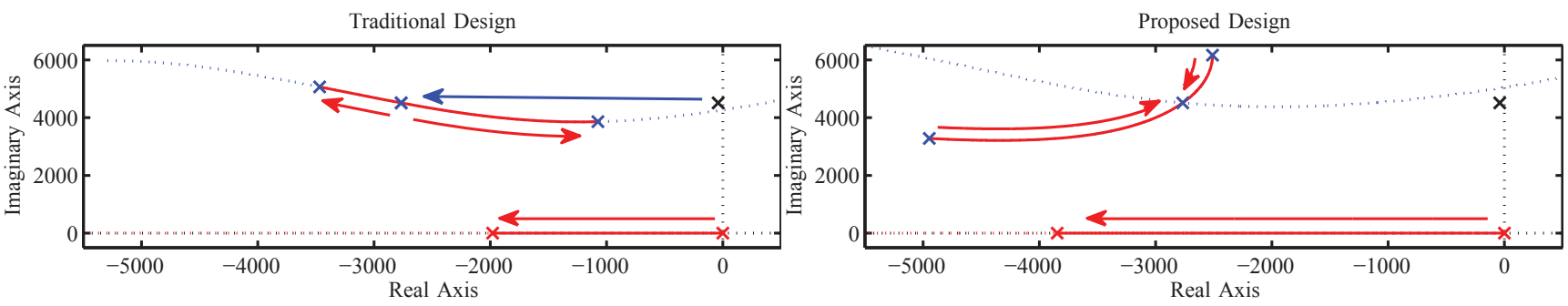

Fig. 3. Root loci of the tracking loop for the traditional controller design and the proposed controller design for the positive imaginary axis only. The image is mirrored on the negative imaginary axis. The black crosses are undamped poles of the open-loop system, blue crosses are the damped poles of the closed-loop system, and the red cross depicts the pole introduced by the tracking controller. In the traditional PVPF design, complex poles are placed at the desired location and the tracking controller displaces these poles from the intended location as the tracking gain is increased. The proposed control design places the damped poles at different strategic locations such that when the desired tracking gain is reached, the PVPF-induced poles converge on the desired (damped) location.
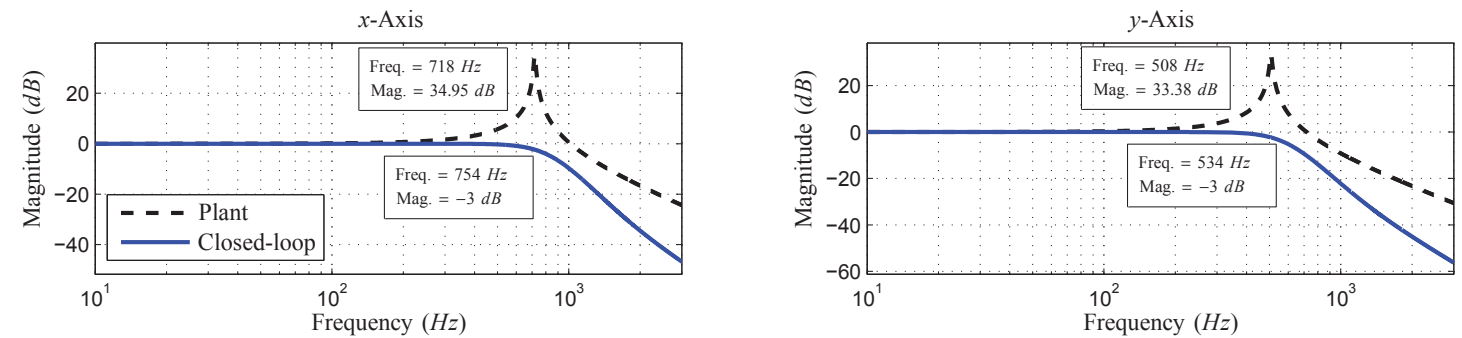

Fig. 4. Bode magnitude plot of the open- and closed-loop system using the second-order model derived from the measured frequency response. The closed-loop model shows the desired flat-band response giving maximum bandwidth. In this the case the $\pm 3 d B$ bandwidth is greater than the resonant frequency for each axis.

is desired. The damping controller should place the poles at two distinct locations. When the tracking controller is applied and the gain increased to the optimal amount, the complex poles should converge at the desired location, as shown if Fig. 3. The damped and tracked system has the following characteristic equation

$$
P(s)=\left(s+\omega_{p}\right)\left(s^{2}+2 \psi \omega_{d} s+\omega_{d}^{2}\right)^{2} .
$$

where $\omega_{p}$ is the real pole introduced by the tracking controller, $\omega_{d}$ is the damped natural frequency of the open-loop system, for lightly damped systems this is approximately equal to $\omega_{n}$, and $\psi$ is the desired damping ratio. Equating this with the denominator in Eqn. (5) gives the equivalent damped and tracked closed-loop system in terms of the desired system variables. This derivation makes the assumption that the damping ratio, $\zeta$, is sufficiently small and can be neglected.

$$
\begin{gathered}
G_{c l}^{n u m}(s)=\frac{\omega_{d}^{4} \omega_{p}\left(s^{2}+\left(4 \psi \omega_{d}+\omega_{p}\right) s+\left(5 \psi \omega_{d}^{2}+\omega_{d}^{2}+4 \psi \omega_{d} \omega_{p}\right)\right)}{5 \psi \omega_{d}^{2}+\omega_{d}^{2}+4 \psi \omega_{d} \omega_{p}} \\
G_{c l}^{d e n}(s)=s^{5}+\left(4 \psi \omega_{d}+\omega_{p}\right) s^{4}+\left(4 \psi^{2} \omega_{d}^{2}+2 \omega_{d}^{2}+4 \psi \omega_{d} \omega_{p}\right) s^{3} \\
+\left(4 \psi \omega_{d}^{3}+4 \psi^{2} \omega_{d}^{2} \omega_{p}+2 \omega_{d}^{2} \omega_{p}\right) s^{2}+\left(\omega_{d}^{4}+4 \psi \omega_{d}^{3} \omega_{p}\right) s+\omega_{d}^{4} \omega_{p} .
\end{gathered}
$$

The aim is to choose $\psi$ and $\omega_{d}$ to achieve a desired amount of damping, and find the $\omega_{p}$ which will give a flat-band response, i.e. $\left|G_{c l}(j \omega)\right|_{d B} \leq 0 \forall \omega \in \mathbb{R}$. The controller parameters will be found by equating the denominator terms of Eqn. (5) with the desired characteristic equation.

$$
\begin{gathered}
\left|G_{c l}(j \omega)\right| \leq 1 \\
\frac{\left|G_{c l}^{n u m}(j \omega)\right|}{\left|G_{c l}^{\text {den}}(j \omega)\right|} \leq 1 \\
\left|G_{c l}^{n u m}(j \omega)\right|^{2} \leq\left|G_{c l}^{\text {den }}(j \omega)\right|^{2} \\
\left|G_{c l}^{\text {den }}(j \omega)\right|^{2}-\left|G_{c l}^{n u m}(j \omega)\right|^{2} \geq 0 \\
\left(\omega^{10}+\left(8 \psi^{2} \omega_{d}^{2}+\omega_{p}^{2}-4 \omega_{d}^{2}\right) \omega^{8}\right. \\
+\left(-16 \psi^{2} \omega_{d}^{4}+8 \psi^{2} \omega_{d}^{2} \omega_{p}^{2}-4 \omega_{d}^{2} \omega_{p}^{2}+6 \omega_{d}^{4}+16 \psi^{4} \omega_{d}^{4}\right) \omega^{6} \\
+\left(6 \omega_{d}^{4} \omega_{p}^{2}+8 \psi^{2} \omega_{d}^{6}-16 \psi^{2} \omega_{d}^{4} \omega_{p}^{2}+16 \psi^{4} \omega_{d}^{4} \omega_{p}^{2}-4 \omega_{d}^{6}\right) \omega^{4} \\
\left.+\left(\omega_{d}^{8}+8 \psi^{2} \omega_{d}^{6} \omega_{p}^{2}-4 \omega_{d}^{6} \omega_{p}^{2}\right) \omega^{2}+\omega_{d}^{8} \omega_{p}^{2}\right) \\
-\left(\frac{\omega_{d}^{4} \omega_{p}}{5 \psi \omega_{d}^{2}+\omega_{d}^{2}+4 \psi \omega_{d} \omega_{p}}\right)^{2}\left(\omega^{4}+\left(6 \psi^{2} \omega_{d}^{2}-2 \omega_{d}^{2}+\omega_{p}^{2}\right) \omega^{2}\right. \\
\left.+\left(25 \psi^{4} \omega_{d}^{4}+\omega_{d}^{4}+16 \psi^{2} \omega_{d}^{2} \omega_{p}^{2}+10 \psi^{2} \omega_{d}^{4}+40 \psi^{3} \omega_{d}^{3} \omega_{p}+8 \psi \omega_{d}^{3} \omega_{p}\right)\right) \geq 0
\end{gathered}
$$

As the system is type 1 , the $\omega^{0}$ terms are equal. Subtracting the $\omega^{0}$ terms and dividing by $\omega^{2}$ gives

$$
\begin{array}{r}
\left(\omega^{8}+\left(8 \psi^{2} \omega_{d}^{2}+\omega_{p}^{2}-4 \omega_{d}^{2}\right) \omega^{6}\right. \\
+\left(-16 \psi^{2} \omega_{d}^{4}+8 \psi^{2} \omega_{d}^{2} \omega_{p}^{2}-4 \omega_{d}^{2} \omega_{p}^{2}+6 \omega_{d}^{4}+16 \psi^{4} \omega_{d}^{4}\right) \omega^{4} \\
+\left(6 \omega_{d}^{4} \omega_{p}^{2}+8 \psi^{2} \omega_{d}^{6}-16 \psi^{2} \omega_{d}^{4} \omega_{p}^{2}+16 \psi^{4} \omega_{d}^{4} \omega_{p}^{2}-4 \omega_{d}^{6}\right) \omega^{2} \\
\left.+\left(\omega_{d}^{8}+8 \psi^{2} \omega_{d}^{6} \omega_{p}^{2}-4 \omega_{d}^{6} \omega_{p}^{2}\right)\right) \\
-\left(\frac{\omega_{d}^{4} \omega_{p}}{5 \psi \omega_{d}^{2}+\omega_{d}^{2}+4 \psi \omega_{d} \omega_{p}}\right)^{2}\left(\omega^{2}+\left(6 \psi^{2} \omega_{d}^{2}-2 \omega_{d}^{2}+\omega_{p}^{2}\right)\right) \geq 0 .
\end{array}
$$



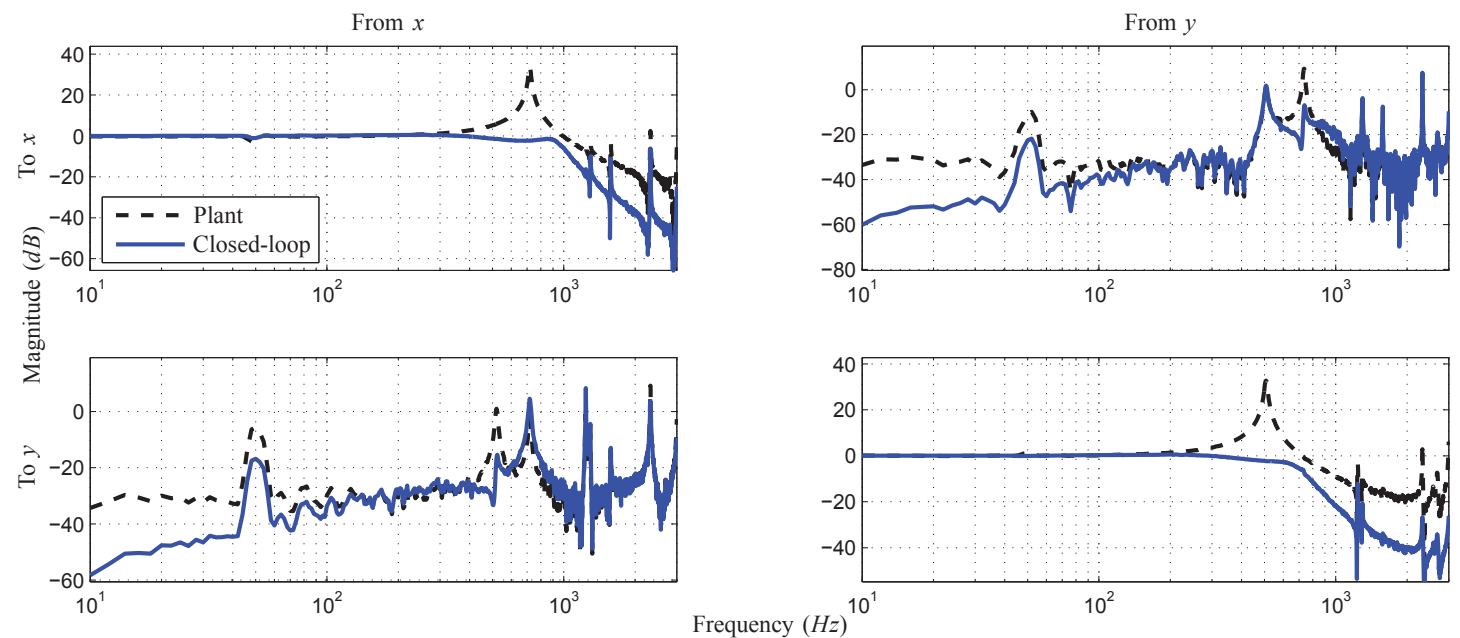

Fig. 5. Closed-loop frequency response of the nanopositioning platform with PVPF controller. As can be seen in the $x$ - (top left) and $y$-axis (bottom right) FRFs, the response is not as flat as the model-based simulation, see Fig. 4. This is due to the effect of the higher order modes not considered in the modelled case.

\section{A. Complex Pole Placement}

For a second-order system with normalized input/output gain, the transfer function is

$$
G(s)=\frac{\omega_{n}^{2}}{s^{2}+2 \zeta \omega_{n} s+\omega_{n}^{2}},
$$

and has magnitude response

$$
|G(j \omega)|=\frac{\omega_{n}^{2}}{\sqrt{\omega^{4}+\left(4 \zeta^{2} \omega_{n}^{2}-2 \omega_{n}^{2}\right) \omega^{2}+\omega_{n}^{4}}} .
$$

For a given a bandwidth of $\pm x d B,|G(j \omega)|$ should not pass through the upper bound on bandwidth, i.e.

$$
|G(j \omega)| \leq 10^{\frac{x}{20}}: \forall \omega \in \mathbb{R}
$$

Substituting Eqn. (12) and rearranging gives

$$
\omega^{4}+\left(4 \zeta^{2} \omega_{n}^{2}-2 \omega_{n}^{2}\right) \omega^{2}+\omega_{n}^{4}-\omega_{n}^{4} \times 10^{\frac{-x}{10}} \geq 0 .
$$

The roots of Eqn. (14) give the frequencies at which $|G(j \omega)|$ crosses $x d B$. If Eqn. (14) has two real and distinct roots, $|G(j \omega)|>x d B$ for some $\omega$, if the roots are real and equal, $|G(j \omega)|=x d B$ for only one value of $\omega$, and if the roots are complex $|G(j \omega)|<x d B \forall \omega$. It is obvious that real and equal roots will provide maximum bandwidth. In this case, the discriminant of Eqn. (14) is equal to zero, i.e.

$$
\begin{array}{r}
\left(4 \zeta^{2} \omega_{n}^{2}-2 \omega_{n}^{2}\right)^{2}+4 \omega_{n}^{4} \times 10^{\frac{-x}{10}}=0 \\
4 \omega_{n}^{4}\left(4 \zeta^{4}-4 \zeta^{2}+10^{\frac{-x}{10}}\right)=0 \\
\Rightarrow \zeta=\sqrt{\frac{4 \pm \sqrt{16-16 \times 10^{\frac{-x}{10}}}}{8}}
\end{array}
$$

Pole placement such that the damping ratio is that given by Eqn. (15) will therefore provide maximum $\pm x d B$ bandwidth relative to the DC gain of a second-order system. For a $\pm 1 d B$ bandwidth, as required in nanopositioning, this gives the damping ratio, $\zeta=0.5227$.

\section{B. Real Pole Placement}

For fixed complex poles, represented by $\psi, \omega_{d}$, Eqn. (10) can be defined as a function of the real pole, $\omega_{p}$, and the frequency, $\omega$, as $H\left(\omega_{p}, \omega\right)$. We choose $\omega_{p}$ such that the following equation is minimized over the bandwidth of interest, i.e. $\omega \in\left[0, \omega_{n}\right]$,

$$
\min \left|\sum_{\omega=0}^{\omega_{n}} H\left(\omega_{p}, \omega\right)\right|
$$

This gives the closed loop frequency response, $G_{c l}(j \omega)$, which is closest to unity gain over the chosen bandwidth.

\section{Controller Synthesis}

With the three system variables defined numerically, the characteristic equation of the system will be of the form

$$
s^{5}+K_{4} s^{4}+K_{3} s^{3}+K_{2} s^{2}+K_{1} s+K_{0} .
$$

Equating this with Eqn. (5) gives the controller parameters as

$$
\begin{array}{r}
2 \gamma \omega_{c}=K_{4}-2 \zeta \omega_{n} \\
\omega_{c}^{2}=K_{3}-\omega_{n}^{2}-2 \zeta \omega_{n} 2 \gamma \omega_{c} \\
k_{t}=K_{0} /\left(\sigma^{2} \omega_{c}^{2}\right) \\
\Gamma_{2}=-\left(K_{2}-2 \zeta \omega_{n} \omega_{c}^{2}-2 \gamma \omega_{c} \omega_{n}^{2}-k t \sigma^{2}\right) / \sigma^{2} \\
\Gamma_{1}=-\left(K_{1}-\omega_{c}^{2} \omega_{n}^{2}-2 \gamma \omega_{c} k_{t} \sigma^{2}\right) / \sigma^{2}
\end{array}
$$

This is used in the following section to derive the controllers for simulation purposes.

\section{Simulation}

Simulations are carried out using the measured frequency response of a two-axis serial kinematic nanopositioner designed and constructed at the EasyLab, University of Nevada, Reno. The stage is driven by a Piezodrive PDL200 $200 \mathrm{~V}$ linear amplifier, and the position measured using a Microsense 

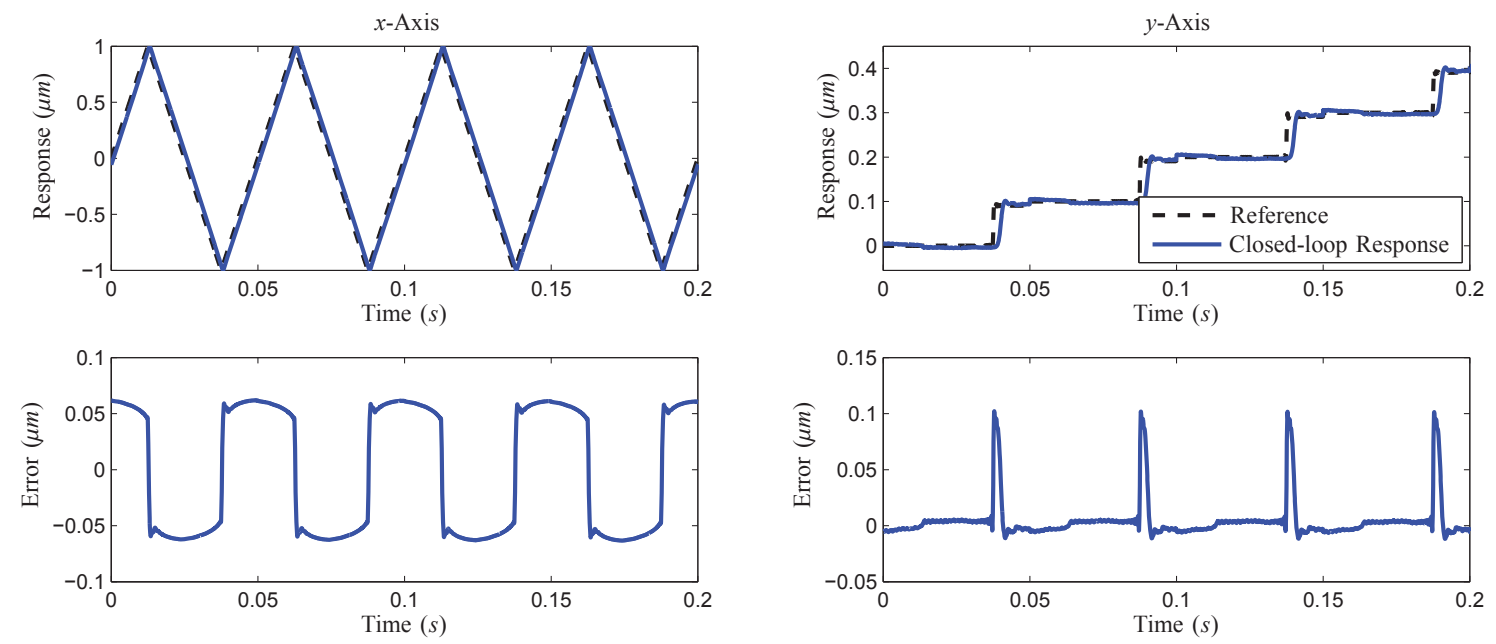

Fig. 6. Plot of the $x$ - and $y$-axis output (top row) and the error relative to the input (bottom row). The magnitude of the $x$-axis error is amplified by the slight time-delay caused by the increased phase of the closed-loop system. By shifting the output by the delay time, a more accurate error signal can be found. For the purpose of simulation, this is not necessary as the $y$-axis input has been delayed by the equivalent time to obtain an accurate scan. Similarly to the $x$-axis case, the maximum $y$-axis error is amplified by the delay induced to account for the delay in the $x$-axis response.

4810 capacitive sensor. The frequency response is measured using an Agilent 35670A Dynamic Signal Analyzer.

The $x$ - and $y$-axis models are derived from the measured frequency response using the discrete time system identification algorithm described in [18]. The continuous time model is then found by bilinear transform giving

$$
\begin{aligned}
G_{x x}(s) & =\frac{2.036 \times 10^{7}}{s^{2}+80.67 s+2.036 \times 10^{7}} \\
G_{y y}(s) & =\frac{1.017 \times 10^{7}}{s^{2}+67.92 s+1.017 \times 10^{7}}
\end{aligned}
$$

\section{A. X-axis Controller}

For a desired damping ratio of 0.5227 , the complex poles of the desired transfer function are set to $-2766.7 \pm 4512.4$. The damped system should then have a maximum resonant peak of no more than $2 d B$. From Eqn. (16), the real pole which gives a flat band response is found to be -3847.0 . This gives the desired characteristic equation as

$$
\begin{aligned}
s^{5}+1.4914 \times 10^{4} s^{4}+ & 1.2923 \times 10^{8} s^{3}+6.4340 \times 10^{11} s^{2} \\
+ & 1.9777 \times 10^{15} s+3.0196 \times 10^{18} .
\end{aligned}
$$

Using Eqn. (18) the controller parameters are calculated, which gives the following controllers

$$
\begin{array}{r}
C_{P V P F x}(s)=\frac{-1.496 \times 10^{4} s+3.097 \times 10^{7}}{s^{2}+1.483 \times 10^{4} s+1.077 \times 10^{8}} \\
C_{t x}(s)=\frac{1377}{s} .
\end{array}
$$

\section{B. Y-axis Controller}

Using the same method as for the $x$-axis design, the complex poles are set to $-1954.9 \pm 3188.4$, the real pole is found to be -2717.3 , giving the desired characteristic equation

$$
\begin{array}{r}
s^{5}+1.0537 \times 10^{4} s^{4}+6.451 \times 10^{7} s^{3}+2.2693 \times 10^{11} s^{2} \\
+4.9286 \times 10^{14} s+5.3164 \times 10^{17} .
\end{array}
$$

Eqn. (18) gives the controllers

$$
\begin{array}{r}
C_{P V P F y}(s)=\frac{-1.052 \times 10^{4} s+1.536 \times 10^{7}}{s^{2}+1.047 \times 10^{4} s+5.363 \times 10^{7}} \\
C_{t y}(s)=\frac{975.1}{s} .
\end{array}
$$

\section{Cross-coupling}

An accurate simulation of a multiple axis scan must account for cross-coupling between the axes. As the nanopositioner used has a serial kinematic design, it is susceptible to significant coupling effects. This is observed in the measured frequency response, Fig. 1. The outputs of the closed-loop system are calculated as

$$
\begin{aligned}
& x=\frac{C_{t x} G_{x x}}{1-G_{x x}\left(C_{P V P F x}-C_{t x}\right)} r_{x}+\frac{G_{x y}}{1-G_{x x}\left(C_{P V P F x}-C_{t x}\right)} r_{y} \\
& y=\frac{G_{y x}}{1-G_{y y}\left(C_{P V P F y}-C_{t y}\right)} r_{x}+\frac{C_{t y} G_{y y}}{1-G_{y y}\left(C_{P V P F y}-C_{t y}\right)} r_{y} .
\end{aligned}
$$

The closed-loop frequency response is shown in Fig. 5. The PVPF controller provides a large reduction of coupling effects at low frequencies.

\section{Raster Scan}

The closed-loop response is simulated using a $\pm 1 \mu \mathrm{m}$, $20 \mathrm{~Hz}$ triangle wave as the $x$-axis input and a $10 \mathrm{~Hz}$ repeating 


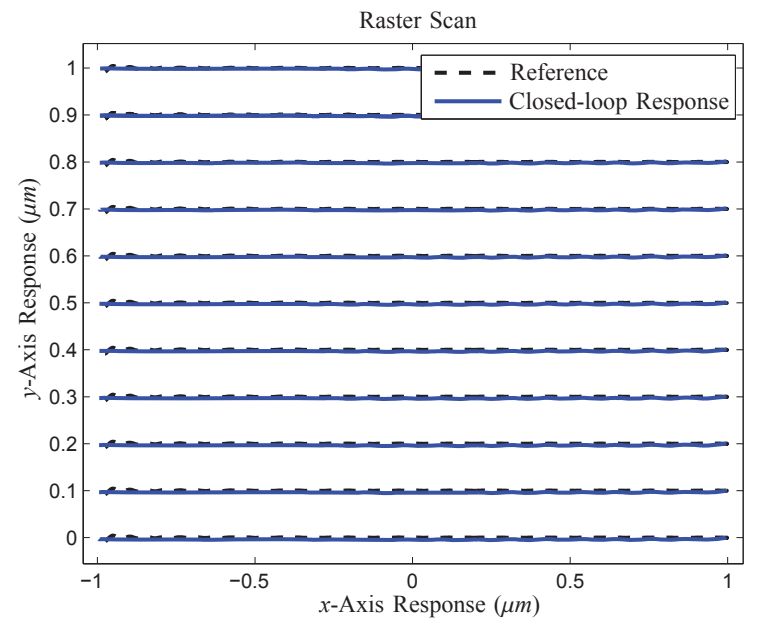

Fig. 7. Simulated raster scan where the $x$-axis input is a $20 \mathrm{~Hz}$ triangle wave with amplitude $\pm 1 \mu \mathrm{m}$, and the $y$-axis input is $10 \mathrm{~Hz}$ stepping function which increases by $0.1 \mu \mathrm{m}$ each period and the step coincides with the lowest point of the $x$-axis trajectory. The phase-lag-induced artifacts present in the full scan during the transition between each consecutive increment of the stepping function have been removed, leaving only the usable scan lines.

step function, with a maximum magnitude of $1 \mu \mathrm{m}$, as the $y$-axis input. The step is placed at

$$
t_{\text {step }}=\frac{i-1}{f_{x 0}}+\frac{3}{4 f_{x 0}}+\frac{\left|\angle G_{x x}^{c l}\left(j \omega_{x 0}\right)\right|}{2 \pi f_{x 0}}, i=1,2, \ldots, n
$$

where $f_{x 0}$ is the base frequency of the $\mathrm{x}$-axis input in $\mathrm{Hz}$, $\angle G_{x x}^{c l}\left(j \omega_{x 0}\right)$ is the phase of the closed-loop $x$-axis frequency response, measured from $r_{x}$ to $x$, at the same frequency, and $n$ is the total number of periods. This coincides with the negative peak of the $x$-axis triangular input. The response of the individual axes are plotted in Fig. 6, and the combined raster scan in Fig. 7. The results are as follows:

\begin{tabular}{|c|c|c|}
\hline & $x$-axis & $y$-axis \\
\hline Resonant Frequency $(\mathrm{Hz})$ & 718 & 508 \\
\hline Bandwidth $\pm 1 d B(\mathrm{~Hz})$ & 468 & 376 \\
\hline Bandwidth $\pm 3 d B(\mathrm{~Hz})$ & 938 & 608 \\
\hline Max. Error $(\mu \mathrm{m})$ & 0.0121 & 0.1022 \\
\hline RMS Error $(\mu \mathrm{m})$ & 0.0045 & 0.0192 \\
\hline
\end{tabular}

Note, the $x$-axis error measurements were calculated using a time-shifted output such that the zero cross-over of both the input and output occur at the same point.

\section{Conclusion}

In this paper, a method for simultaneously optimizing the parameters of a PVPF damping controller and an integral tracking controller for high-precision positioning applications is provided. The model-based simulations show a flat band response with high bandwidth, perfectly suited to high-speed scanning. The frequency response based simulations exhibit the effect of the systems higher order dynamics but maintain a largely flat response and similarly high bandwidth. Despite the example systems tendency to exhibit cross-coupling effects, the controller significantly reduces them. This method is, therefore, ideal for improving the positioning bandwidth of existing nanopositioners.

\section{REFERENCES}

[1] A. Preumont, Vibration Control of Active Structures: An Introduction. Dordrecht: Kluwer, 1997.

[2] S. S. Aphale, A. J. Fleming, and S. O. R. Moheimani, "Integral resonant control of collocated smart structures," Smart Materials and Structures, vol. 16, pp. 439 - 446, February 2007.

[3] A. Preumont, B. de Marneffe, A. Deraemaeker, and F. Bossens, "The damping of a truss structure with a piezoelectric transducer," Computers and Structures, vol. 86, pp. 227 - 239, 2008.

[4] S. S. Aphale, B. Bhikkaji, and S. R. Moheimani, "Minimizing scanning errors in piezoelectric stack-actuated nanopositioning platforms," IEEE Transactions on Nanotechnology, vol. 7, no. 1, pp. 79-90, 2008.

[5] J. L. Fanson and T. K. Caughey, "Positive position feedback control for large space structures," AIAA Journal, vol. 28, no. 4, pp. 717 724, 1990.

[6] B. Bhikkaji, M. Ratnam, A. J. Fleming, and S. O. R. Moheimani, "High-performance control of piezoelectric tube scanners," IEEE Transactions on Control Systems Technology, vol. 15, no. 5, pp. 853 - 866, September 2007.

[7] E. Pereira, S. S. Aphale, V. Feliu, and S. O. R. Moheimani, "Integral resonant control for vibration damping and precise tip-positioning of a single-link flexible manipulator," IEEE / ASME Transactions on Mechatronics, vol. 16, no. 2, pp. 232 - 240, April 2011.

[8] H. Numasato and M. Tomizuka, "Settling control and performance of a dual-actuator system for hard disk drives," IEEE/ASME Transactions on Mechatronics, vol. 8, no. 4, pp. 431-438, 2003.

[9] P. P. Friedmann and T. A. Millott, "Vibration reduction in rotorcraft using active control: A comparison of various approaches," Journal of Guidance, Control and Dynamics, vol. 18, no. 4, pp. 664 - 673, 1995.

[10] A. J. Fleming, "Nanopositioning system with force feedback for highperformance tracking and vibration control," IEEE / ASME Transactions on Mechatronics, vol. 15, no. 3, pp. 433-447, 2010.

[11] A. J. Fleming, S. S. Aphale, and S. O. R. Moheimani, "A new method for robust damping and tracking control of scanning probe microscope positioning stages," IEEE Transactions on Nanotechnology, vol. 9, no. 4, pp. 438 - 448, July 2010.

[12] A. Ferreira and S. S. Aphale, "A survey of modeling and control techniques for micro- and nanoelectromechanical systems," IEEE Transactions on Systems, Man and Cybernetics, Part C: Applications and Reviews, vol. 41, pp. $350-364,2011$.

[13] A. Sebastian, A. Pantazi, R. Moheimani, H. Pozidis, and E. Eleftheriou, "Achieving sub-nanometer precision in a mems storage device during self-servo write process," IEEE Transactions on Nanotechnology, vol. 7, no. 5, pp. 586 - 595, September 2008.

[14] S. R. Moheimani, "Invited review article: Accurate and fast nanopositioning with piezoelectric tube scanners: Emerging trends and future challenges," Review of Scientific Instruments, vol. 7, no. 7, 2008.

[15] S. Devasia, E. Eleftheriou, and S. O. R. Moheimani, "A survey of control issues in nanopositioning," IEEE Transactions on Control Systems Technology, vol. 15, no. 4, pp. 689 - 703, July 2007.

[16] A. Bazaei, Y. K. Yong, S. O. R. Moheimani, and A. Sebastian, "Tracking of triangular references using signal transformation for control of a novel afm scanner stage," IEEE Transactions on Control Systems Technology, vol. 20, no. 2, pp. 453-464, 2012.

[17] M. Namavar, A. J. Fleming, M. Aleyaasin, K. Nakkeeran, and S. S. Aphale, "An analytical approach to integral resonant control of secondorder systems," in press, IEEE Transactions on Mechatronics, 2013.

[18] T. McKelvey, H. Akcay, and L. Ljung, "Subspace based multivariable system identification from frequency response data," IEEE Tansaction on Automatic Control, vol. 41, no. 7, pp. 960-979, 1996. 